\title{
Presupuesto, gasto social y desarrollo municipal
}

El Presupuesto General de la Nación (PGN) para 1998 ha sido probablemente el más debatido de toda la década de 1990. El aspecto más polémico ha estribado en la discusión legislativa y la subsecuente negociación con el poder ejecutivo sobre las asignaciones para los gobiernos municipales. Este tema llegó a empantanar la aprobación del presupuesto de 1998, al grado de aprobarse cuando casi finalizaba el primer mes del año. La propuesta de la mayor parte de alcaldías y del principal partido de oposición, el FMLN, contemplaba asignar un 6 por ciento del presupuesto general de la nación, es decir, cerca de 1,013 millones de colones, mientras que la asignación inicial propuesta por el gobierno a través del Ministerio de Hacienda, apenas alcanzaba el 2 por ciento del presupuesto, equivalente a 330 millones de colones.

Al examinar las asignaciones, y sin pretender negar la importancia del fortalecimiento municipal para el desarrollo, no puede dejar de mencionarse que su discusión desvió la atención de otros aspectos igualmente cuestionables. Primero, la caída de la participación porcentual del gasto social en el Presupuesto General de la Nación; segundo, el surgimiento de nuevos rubros del gasto público provenientes de la "modernización" del Estado y, tercero, el sensible incremento de las asignaciones para el servicio de la deuda externa. Es notable también que se ha reducido la participación de los rubros de defensa y seguridad pública, los cuales desde la década de los ochenta, y hasta mediados de los noventa, fueron los principales destinos de los incrementos del Plan.
Por el lado de los ingresos, destaca el hecho de que estos no han logrado incrementarse lo suficiente como para erradicar el déficit fiscal, y ni siquiera para contener su expansión. Afortunadamente, para 1998 se esperan importantes ingresos fiscales provenientes de la transferencia de los fondos resultantes de la venta de las empresas estatales de servicios públicos que permitirán reducir el déficit fiscal y el endeudamiento.

En lo que sigue, se analiza la evolución del Plan General de la Nación, no sólo respecto a las asignaciones municipales, sino también al desarrollo social, a la modemización del Estado y al servicio de la deuda. Asimismo, se revisan algunos características de la estrategia de financiamiento del Plan, especialmente la estructura tributaria y la venta de empresas públicas.

El Plan General de Nación ha mostrado una tendencia alcista durante toda la década de 1990, pese a que durante la misma los gobiernos de turno han pregonado la importancia de la austeridad fiscal como medio para la estabilidad macroeconómica. Lamentablemente, los incrementos no se han destinado a fortalecer las áreas más descuidadas y donde más se justifica la intervención del Estado, como lo es la atención de las necesidades básicas.

En conjunto, las ramas consideradas dentro del gasto social (educación, salud, vivienda y trabajo) disminuyeron su participación en el total del presupuesto. En 1996 y 1997 se destinaron 24.35 y 25.1 por ciento para gasto social, respectivamente, mientras que en 1998 se espera que el gasto social alcan- 
ce únicamente el 24.5 por ciento del total del presupuesto. Este porcentaje resulta de sumar los porcentajes asignados a educación (16.1 por ciento), salud (7.9 por ciento), vivienda ( 0.2 por ciento) y trabajo ( 0.3 por ciento). Esta política es una clara incoherencia con el Plan de Desarrollo Social ofrecido por el Presidente Armando Calderón Sol, en el cual ofrecía incrementar el presupuesto para el área social hasta un 50 por ciento para 1999.

El gasto social en el Plan General de Nación relrocedió a los niveles de hace 2 años. Dentro de este comportamiento, lo más cuestionable es la reducción del porcentaje asignado a la salud. Desde 1996 a 1998, ćste ha experimentado constantes reducciones de su participación porcentual. En 1996, cl presupuesto de salud fue de 9.23 por ciento del tolal; en 1997, de 8.6 por ciento, y para 1998 representará tan sólo un 7.9 por ciento. El presupuesto para educación apenas se incrementó de 16 a 16.1 por ciento del total del PGN. Al analizar los componentes trabajo y vivienda, destaca que estos se mantuvieron prácticamente en los mismos niveles porcentuales de 1997, pero al considerar las asignaciones para 1996 sí pueden notarse pequeños cambios. La asignación porcentual para el ramo de vivienda disminuyó de 0.3 a 0.2 por ciento del total, y la de trabajo y previsión social fue de 0.21 a 0.3 . Es decir, que lo ganado en el ámbito de trabajo prácticamente se perdió en el ramo de vivienda. Aunque no implicó una reducción de su participación porcentual, el ramo de vivienda en realidad sufrió un recorte de 1.2 millones de colones, pese a que la vivienda es una de las necesidades sociales más ingentes. Esta es una muestra palmaria del desinterés gubernamental en la promoción del desarrollo social.

Un segundo punto cuestionable del nuevo presupuesto es el surgimiento de gastos extraordinarios hasta por un valor de 4.1 por ciento del presupuesto total. Asî han surgido rubros como "Estabilidad y fomento económico", "Compensación por méritos", "Modernización de la Administración Fiscal" y "Financiamiento al FIS", que han venido a absorber cerca de un 4.1 por ciento del presupuesto total de la nación, mucho más de lo que se redujo a los rubros social, policial, defensa nacional y servicio de la deuda total, que totaliza apenas un 1.3 por ciento del total del presupuesto.

A excepción de la partida dedicada al FIS, las restantes están muy relacionadas con los fines de modernización del Estado en que los dos últimos gobiernos se han empeñado, y que persiguen redu- cir el tamaño del Estado para que ésta atienda sólo funciones esenciales. Así, se proponen gastos para mejorar las funciones del Estado en el ámbito del fomento económico y de la administración fiscal y la reducción del tamaño del Estado, para lo cual se ofrecen programas compensatorios que fomenten el retiro de los empleados estatales, práctica que se remonta a cuando menos 3 años atrás. Implícitamente, la "modernización" persigue también la reducción de las presiones financieras y la inestabilidad económica que surge de la existencia de un Estado demasiado grande pero, paradójicamente, al menos para 1998 los esfuerzos de modernización causarán precisamente un efecto inverso pues provocarán un incremento del presupuesto general equivalente a un 4 por ciento, si se excluye el aporte FIS.

Un tercer aspecto de las asignaciones presupuestarias por destacar es el incremento del servicio de la deuda externa. Aunque en su conjunto el servicio de la deuda total ha perdido peso en el total del presupuesto, el servicio de la deuda externa se incrementaría notablemente entre 1997 y 1998, pasando de 8.8 a 9.7 por ciento, respectivamente. La reducción del servicio total se explica solamente por la reducción del servicio de la deuda interna. Para 1998, el servicio de la deuda externa podría llegar a mejorar su participación en el total del presupuesto aun por encima de lo que lo haría el ramo de educación. Como se mencionó, el presupuesto para educación únicamente se incrementó de 16 a 16.1 por ciento, un total de 0.1 por ciento, mientras que el servicio de la deuda recibió un 0.9 por ciento del total del presupuesto más que en 1997. Esto resulta especialmente cuestionable si consideramos que se ha reformado el sistema tributario, precisamente para reducir las presiones fiscales y el nivel de endeudamiento del país.

Es importante reseñar aquí que los rubros de seguridad ciudadana y defensa nacional, al igual que el área social, también han experimentado sucesivas reducciones en su participación en el PGN. La seguridad pública -que recibio 9.1 por ciento del total del presupuesto en 1996 - recibira 7.8 por ciento del total del presupuesto en 1998; mientras que la defensa nacional -que obtenía el 6 por cientorecibirá 5.2 por ciento. El órgano judicial, por su parte, habría experimentado un crecimiento de su participación, pues de obtener un 5 por ciento del presupuesto en 1997 obtendrá el 6.1 por ciento en 1998. Asimismo, el servicio de la deuda interna 


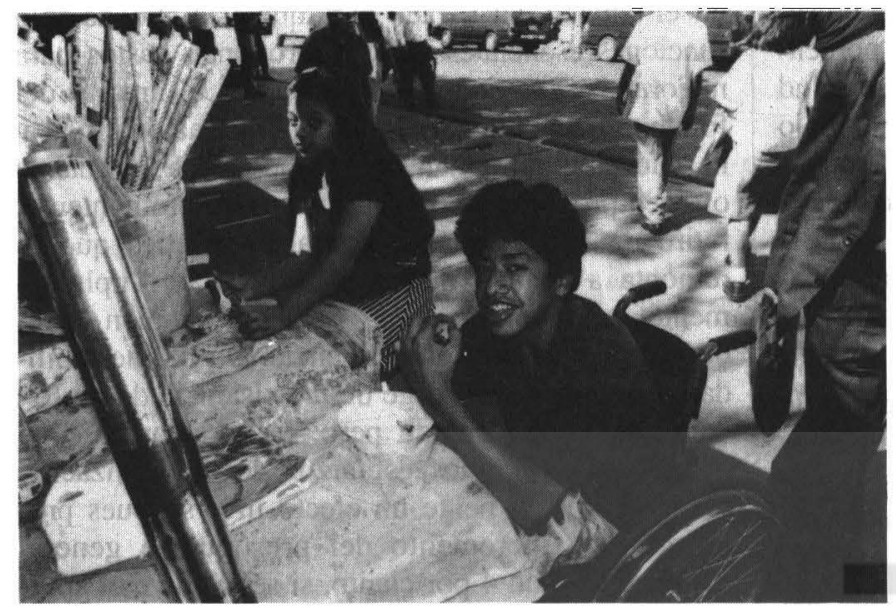

que entre ambos aportan un 84.2 por ciento del total de los ingresos tributarios; otros impuestos han desaparecido (exportaciones y al patrimonio) o se han contraído substancialmente (importaciones). Para 1998 se espera que el impuesto a las importaciones reduzca su participación en los ingresos tributarios; así, representaría sólo 8.1 por ciento del mencionado total, cuando en 1997 representó el 10.3 por ciento.

Por otra parte, es importante mencionar que para 1998 se proyecta financiar el presupuesto con parte de los fondos provenientes de la privatización de las empresas distribuidoras de energía eléc-

también experimentó una reducción, ya que de percibir el 8.4 por ciento del presupuesto en 1997 percibirá el 7.4 por ciento en 1998, de esta manera permitirá reducir - pese al incremento del servicio de la deuda externa - el servicio de la deuda total.

No es ocioso revisar en este punto cual será el comportamiento de las finanzas públicas de cara a las exigencias del nuevo presupuesto, especialmente porque éste muestra una clara tendencia expansiva que presiona para la permanencia del déficit fiscal. Para 1998 se estima que el PGN se incrementará en 10 por ciento y el déficit fiscal alcanzará el 1.9 por ciento del Producto Interno Bruto. Según apreciaciones del Banco Central de Reserva, esta siluación obedece grandemente a una deficiente gestión de las finanzas públicas por parte del Ministerio de Hacienda, tanto en lo concerniente a la recaudación como en el endeudamiento. En primer lugar, se señala su incapacidad para elevar significativamente la carga tributaria, la cual habría sido apenas del 10.6 por ciento para $1997 \mathrm{y}$, en segundo lugar, la creciente política de endeudamiento externo y el consecuente drenaje de las finanzas públicas debido al elevado servicio de la deuda externa. Lo cierto es que estas defíciencias se atribuyen no sólo al Ministerio de Hacienda, sino a todo el conjunto de reformas tributarias de la década de los noventa.

En lo tocante a los ingresos, puede mencionarse que para 1998 se profundizó la dependencia de los ingresos tributarios del Impuesto al Valor Agregado (IVA), el cual llegó a representar más del 56 por ciento del total de ingresos tributarios. Si se agregan el IVA y el impuesto sobre la renta, se tiene trica. Así se contempla que de los $\mathbf{5 5 0}$ millones de dólares obtenidos en la subasta de las distribuidoras de energía eléctrica, 320 millones sean transferidos al fondo de la nación. Esto representaría cerca del 16.5 por ciento del total del presupuesto de 1998 . De la misma manera, se planea dedicar el producto de la venta de la empresa telefónica estatal, a subastarse a mediados de año, al financiamiento de programas de instituciones públicas.

El endeudamiento externo, por su parte, ha experimentado una marcada tendencia a incrementarse durante los últimos años, manteniéndose como una alternativa para financiar el déficil fiscal. Así, entre 1995 y 1998 se espera su ascenso a 36 por ciento, por lo cual no es de extrañar que el servicio de la deuda externa ha venido absorbiendo cada vez mayores proporciones del presupuesto de la nación, tal como se evidenció anteriormente.

No puede dejar de mencionarse en este comentario el tema del financiamiento a los gobiernos municipales, el área más espinosa del PGN de 1998. Tanto el Presidente de la República como personeros del Ministerio de Hacienda han insistido en que no existían los recursos financieros para otorgar el 6 por ciento del presupuesto a los gobiernos municipales. Por su parte, representantes de la oposición argumentaron que la citada asignación ya había sido aceptada por el mismo presidente antes que el PGN fuese presentado a la Asamblea Legislativa.

Lo cierto es que aunque el gobierno afirma estar a favor de la descentralización y el fortalecimiento municipal -que es un objetivo declarado en sus propuestas de gobierno-, en la práctica pretendió 
bloquear iniciativas que podrían contribuir significativamente a mejorar la capacidad de gestión del desarrollo a nivel municipal.

En síntesis, los aspectos más destacables del presupuesto para 1998 por el lado del gasto son la reducción del gasto social, el incremento del financiamiento a las municipalidades y la inclusión de gastos contingenciales destinados a la modernización del Estado. Por el lado de los ingresos, destaca el hecho de que parte del presupuesto, y del déficil fiscal, podrá ser financiado temporalmente por la entrada de recursos provenientes de la venta de activos del Estado (distribuidoras de energía eléctrica y empresa de telecomunicaciones).

Como ha sido costumbre, el Presupuesto General de la Nación mantiene una franca tendencia ascendente, pero sin inclinarse hacia los sectores vinculados con la función social del Estado. Resulta ilustrativo que, para 1998, los rubros que recibirían los mayores incrementos serían los de educación y el servicio de la deuda externa, lo cual sugiere que el servicio de la deuda externa se encuentra en franca competencia con los rubros del gasto social, las asignaciones para las municipalidades o con cualquier otra función del Estado.

La composición del presupuesto revela, nuevamente, que uno de los mayores vacíos de los programas de gobierno se encuentra en el área de fomento al desarrollo social. Por ejemplo, aunque no es necesario argumentar en torno a la insuficiencia y constante deterioro del sistema nacional de salud, en la práctica, la asignación presupuestaria para esta rama resulta francamente incoherente, no sólo con las necesidades del país, sino también con el discurso oficial sobre el desarrollo social.
La elevación del financiamiento para las municipalidades representa una importante oportunidad para fortalecer la descentralización y el desarrollo local. En buena medida, este incremento se debió a los planteamientos y negociaciones desarrolladas entre los poderes legislativo y ejecutivo, lo cual mueve a pensar que en ese mismo nivel debería de ventilarse también la inverosímil política del gobierno de reducir el gasto social, que por ahora no encuentra ninguna resistencia en el ámbito legislativo.

Las implicaciones de la modernización del Estado sobre el presupuesto de 1998 han sido ambiguas. Por una parte, se dedica un 4 por ciento del presupuesto para estas tareas pero, por el otro, existe la posibilidad de financiamiento hasta del 16 por ciento para el citado presupuesto. Con todo, al menos para 1998, la modernización del Estado parece que rendirá sus dividendos en términos de reducción de las presiones hacia el déficit fiscal.

Pese a que el Presupuesto General de la Nación para 1998 fue elaborado bajo una nueva correlación de fuerzas en la Asamblea Legislativa, el gasto social no pudo incrementar su participación porcentual. En cambio, las asignaciones para los gobiernos municipales sí fueron objeto de intensas negociaciones, aunque en realidad este rubro no está tan directamente ligado con la satisfacción de las necesidades básicas como lo están los rubros de educación y salud, por ejemplo.

Lo anterior sugiere que no solamente el gobierno de ARENA desestima la importancia de incrementar el gasto social, también los sectores de la oposición legislativa adoptaron esa postura en el momento de negociar el presupuesto. 


\section{Cuadro 1}

Asignaciones presupuestarias y composición de los ingresos tributarios y por privatizaciones (1998)

\begin{tabular}{|c|c|c|c|}
\hline \multirow[b]{2}{*}{ Rubros } & \multicolumn{3}{|c|}{ Porcentajes del total del presupuesto } \\
\hline & 1996 & 1997 & 1998 \\
\hline Desarrollo social & 24.35 & 25.1 & 24.5 \\
\hline Educación & 14.60 & 16.0 & 16.1 \\
\hline Salud & 9.23 & 8.60 & 7.9 \\
\hline Vivienda & 0.31 & 0.20 & 0.20 \\
\hline Trabajo y prev. social & 0.21 & 0.30 & 0.30 \\
\hline Servicio de la deuda & 17.9 & 17.2 & 17.1 \\
\hline Externa & n.d. & 8.8 & 9.7 \\
\hline Interna & n.d. & 8.4 & 7.4 \\
\hline \multicolumn{4}{|l|}{ Gastos defensa, seguridad } \\
\hline pública y admon. justicia & 20.1 & 19.70 & 19.1 \\
\hline Seguridad Pública & 9.10 & 8.90 & 7.8 \\
\hline Defensa Nacional & 6.00 & 5.80 & 5.2 \\
\hline Organo judicial & 5.00 & 5.00 & 6.1 \\
\hline \multicolumn{4}{|l|}{$\begin{array}{l}\text { Asignaciones a los } \\
\text { gobiernos municipales }\end{array}$} \\
\hline Nuevos rubros & s.a. & s.a. & 4.1 \\
\hline Estabilidad y fomento económico & s.a. & s.a. & 1.0 \\
\hline Compensación por méritos & s.a. & s.a. & 2.3 \\
\hline Modernización fiscal & s.a. & s.a. & 0.7 \\
\hline \multicolumn{4}{|l|}{ Financiamiento al FIS } \\
\hline & Porcentaj & total de & s tributarios \\
\hline Rubros & 1996 & 1997 & 1998 \\
\hline Impuesto sobre la renta & 51.8 & 27.9 & 28.1 \\
\hline IVA & 26.8 & 52.9 & 56.2 \\
\hline Importaciones & 14.0 & 10.8 & 8.1 \\
\hline Exportaciones & 0 & 0 & 0 \\
\hline Consumo & 6.2 & 7.1 & 6.4 \\
\hline Otros & 1.2 & 1.3 & 1.2 \\
\hline
\end{tabular}

n.d.: No disponible.

s.a.: Sin asignación.

Fuente: Anteproyecto del Presupuesto General de la Nación y revistas del Banco Central de Reserva.

Luis E. Romano 